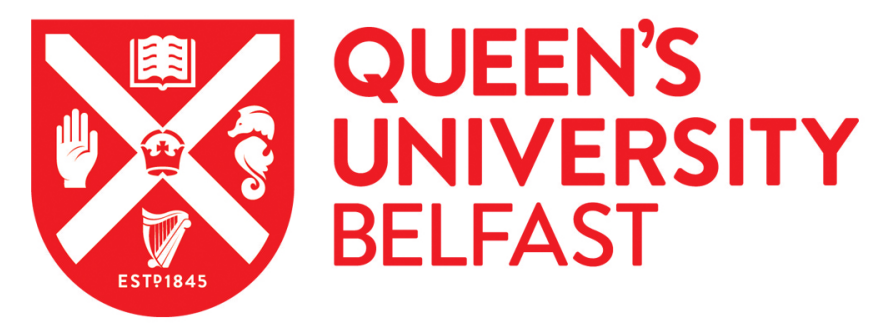

\title{
The Egalitarian Shapley Value: A generalization based on coalition sizes
}

\author{
Borkotokey, S., Choudhury, D., Kumar, R., \& Sarangi, S. (2020). The Egalitarian Shapley Value: A \\ generalization based on coalition sizes. Annals of Operations Research, 301, 55-63. \\ https://doi.org/10.1007/s10479-020-03675-9
}

\section{Published in:}

Annals of Operations Research

\section{Document Version:}

Peer reviewed version

\section{Queen's University Belfast - Research Portal:}

Link to publication record in Queen's University Belfast Research Portal

\section{Publisher rights}

Copyright 2020 Springer. This work is made available online in accordance with the publisher's policies. Please refer to any applicable terms of use of the publisher.

\section{General rights}

Copyright for the publications made accessible via the Queen's University Belfast Research Portal is retained by the author(s) and / or other copyright owners and it is a condition of accessing these publications that users recognise and abide by the legal requirements associated with these rights.

Take down policy

The Research Portal is Queen's institutional repository that provides access to Queen's research output. Every effort has been made to ensure that content in the Research Portal does not infringe any person's rights, or applicable UK laws. If you discover content in the Research Portal that you believe breaches copyright or violates any law, please contact openaccess@qub.ac.uk. 


\title{
The Egalitarian Shapley Value: A generalization based on coalition sizes
}

\author{
Dhrubajit Choudhury* $^{*}$ Surajit Borkotokey ${ }^{\dagger}$ Rajnish Kumar ${ }^{\ddagger}$ Sudipta Sarangi ${ }^{\S}$
}

\begin{abstract}
In designing solution concepts for cooperative games with transferable utilities, consolidation of marginalism and egalitarianism has been widely studied. The $\alpha$-Egalitarian Shapley value is one such solution that combines the Shapley value and the Equal Division rule, the two most popular extreme instances of marginalism and egalitarianism respectively. This value gives the planner the flexibility to choose the level of marginality for the players by varying the convexity parameter $\alpha$. In this paper, we define the Generalized Egalitarian Shapley value that gives the planner more flexibility in choosing the level of marginality based on the coalition size. We then provide two characterizations of the Generalized Egalitarian Shapley value.
\end{abstract}

Keywords: Shapley value; Equal Division rule; Solidarity; Egalitarian Shapley value.

MSC(2010): 91A12; JEL: C71, D60.

\section{Introduction}

Values in cooperative games with transferable utilities (TU games) prescribe allocations of players' cooperative outcomes based on different intuitive and fairness axioms. In this paper, we introduce the Generalized Egalitarian Shapley value for TU games that consolidates marginalism and egalitarianism on the basis of the size of coalitions. Many of the values found in the literature revolve around these two properties of marginalism and egalitarianism. Important examples are the Shapley value [15] and the Equal division (ED) rule respectively. Values that are designed to consolidate marginalism and egalitarianism extend solidarity to the weak or non-productive players in a group. The $\alpha$-Egalitarian Shapley value due to [10] is a convex combination of the Shapley value and the Equal Division rule determined by the convexity parameter $\alpha \in[0,1]$. This value gives the planner the flexibility to choose the level of solidarity in the allocation by varying the parameter $\alpha \in[0,1]$. In this paper, we provide a generalization of this value that takes coalition size into account, especially by considering situations where small groups are egalitarian while larger groups follow marginalism.

In our society, egalitarianism is often observed in small coalitions while marginalism is observed in large coalitions. Take the typical start-up or the so-called "flat organizations" that can be found in hitech hubs such as Silicon Valley. At the beginning, these firms are small, lack hierarchical structures and follow an egalitarian compensation scheme for the small set of founding members. As the organization grows, new employees are often paid according to their contribution to the firm. This type of norm is also visible in resource allocation that occurs between in-groups and out-groups. For instance, Fehr et al. 8] provide evidence for in-group egalitarianism in children and argue that it has deep roots in human development 1 It is observed that in small sized coalitions within a community or a tribe or clan, players are more friendly, homogenous and socially involved. As a result there is more egalitarian resource sharing among them. However, when more people enter into the coalition making it sufficiently large and

\footnotetext{
*Department of Mathematics, Dibrugarh University, Dibrugarh, Assam-786004, India; Email:dhrubajit@gmail.com

$\dagger$ Department of Mathematics, Dibrugarh University, Dibrugarh, Assam-786004, India; Email: surajitbor@yahoo.com

‡Economics Group, Queen’s Management School, Queen’s University Belfast, UK;Email: rajnish.kumar@qub.ac.uk

$\S$ Department of Economics, Virginia Tech, USA; Email: ssarangi@vt.edu

${ }^{1} \mathrm{He}$ et al. 9] find similar patterns in reciprocity and cooperation in tightly knit clans in rural China.
} 
heterogeneous, productive players prefer not to share their earnings equally with non-productive or less productive players.

Motivated by the above discussion, this paper introduces a new class of values, termed the Generalized $\boldsymbol{\alpha}$-Egalitarian Shapley values or simply the $\boldsymbol{\alpha}$-GES values, that incorporate the level of egalitarianism for players based on the size of coalitions. We provide two characterizations of this class of values. The $\boldsymbol{\alpha}$-GES value is defined on a decomposition of the game space with respect to a vector $\boldsymbol{\alpha}=\left(\alpha_{1}, \cdots, \alpha_{n}\right) \in[0,1]^{n}$, where these $\alpha_{k} \mathrm{~s}$, one for each coalition size are non-decreasing and correspond to the size of the coalitions. Thus, it gives the planner more flexibility in deciding the level of solidarity for players. Along with the standard axioms of TU games, we characterize the $\boldsymbol{\alpha}$-GES value using the axiom: null player in a nonnegative environment property. This axiom requires non-negative allocation to a null player when every coalition containing this player has a non-negative worth. The null player in a non-negative environment property is a weaker version of the null player in a productive environment property introduced in [4]. The null player in a productive environment property shows solidarity to the null player if the grand coalition generates non-negative worths. The null player in a non-negative environment property, on the other hand, shows solidarity only when all the coalitions that include the null player generate non-negative worths.

The rest of the paper proceeds as follows. In Section 2 we present the definitions and results necessary for the development of the paper. In Section 3 we introduce and characterize the $\boldsymbol{\alpha}$-GES value. Section 4 concludes.

\section{Preliminaries}

Let $N=\{1,2, \ldots, n\}$ be the player set with $n$ players. Denote the size of a coalition $S$ by the corresponding lower case letter $s$. To simplify notation, we write $S \cup i$ for $S \cup\{i\}$ and $S \backslash i$ for $S \backslash\{i\}$ for each $S \subseteq N$ and $i \in N$. Also we denote by the small letters $s, t$ etc., the size of coalitions $S, T$ etc., respectively. A cooperative game with transferable utilities or simply a TU game is a pair $(N, v)$ where the function $v: 2^{N} \rightarrow \mathbb{R}$ is such that $v(\emptyset)=0$. For each $S \subseteq N, v(S)$ denotes the worth of the coalition $S$. If the player set $N$ is fixed, we represent a TU game by $v$ only. Denote by $v_{0}$ the null game, defined as $v_{0}(S)=0$ for all $S \subseteq N$. The class of all TU games over the player set $N$ is denoted by $G(N)$ which forms a vector space of dimension $2^{n}-1$ under the standard addition and scalar multiplications of set functions. For every coalition $S \subseteq N$ with $S \neq \emptyset$, the game $e_{S}: 2^{N} \rightarrow \mathbb{R}$ given by,

$$
e_{S}(T)= \begin{cases}1, & \text { if } T=S \\ 0, & \text { otherwise }\end{cases}
$$

is called the identity game. The identity games form a standard basis of $G(N)$. Any $v \in G(N)$ can be uniquely represented as $v=\sum_{S \neq \emptyset} v(S) e_{S}$. A value on $G(N)$ is a function $\Phi$ that assigns a payoff vector $\Phi(v) \in \mathbb{R}^{n}$ to each $v \in G(N)$ for a fixed player set $N$. The Shapley value (representing marginalism) [15] and the Equal division (ED) rule (representing egalitarianism) are respectively given by

$$
\begin{gathered}
\Phi_{i}^{S h}(v)=\sum_{S \subseteq N \backslash i} \frac{s !(n-s-1) !}{n !} v(S \cup i)-v(S) . \\
\Phi_{i}^{E D}(v)=\frac{v(N)}{n} .
\end{gathered}
$$

For $\alpha \in[0,1]$, the $\alpha$-Egalitarian Shapley value due to [10, which is a convex combination of these, is given by

$$
\Phi_{i}^{\alpha-E S}(v)=\alpha \Phi_{i}^{S h}(v)+(1-\alpha) \Phi_{i}^{E D}(v),
$$

where the parameter $\alpha \in[0,1]$ makes the reconciliation between marginalism and egalitarianism. Various axiomatizations of the Shapley value, the Equal Division rule and the $\alpha$-Egalitarian Shapley value can be found in the literature (see [1, 2, 3, 4, 11, 15, 16, 17, 18).

Next we provide some definitions and list some of the axioms that have been used to characterize these three values, focusing on the ones most relevant to us. 
Definition 1. A player $i \in N$ is a null player in $v$ if $v(S \cup i)=v(S)$ for every coalition $S \subseteq N$.

Definition 2. A player $i \in N$ is a nullifying player in $v$ if $v(S)=0$ for every coalition $S$ with $i \in S$.

Definition 3. Two players $i, j \in N$ are called symmetric with respect to the game $v$ if for all $S \subseteq$ $N \backslash\{i, j\}, v(S \cup i)=v(S \cup j)$.

The axioms for a value $\Phi: G(N) \rightarrow \mathbb{R}^{n}$ are listed below.

Axiom 1. Efficiency: For any $v \in G(N)$, we have $\sum_{i \in N} \Phi_{i}(v)=v(N)$.

Axiom 2. Null player property: For each $v \in G(N)$, we have $\Phi_{i}(v)=0$ for every null player $i \in N$ in $v$.

Axiom 3. Nullifying player property: For each $v \in G(N)$, we have $\Phi_{i}(v)=0$ for every nullifying player $i \in N$ in $v$.

Axiom 4. Null game property: For all $i \in N, \Phi_{i}\left(v_{0}\right)=0$.

Axiom 5. Symmetry: For every pair of symmetric players $i, j \in N$ with respect to the game $v \in G(N)$, we have $\Phi_{i}(v)=\Phi_{j}(v)$.

Axiom 6. Linearity: For all $u, w \in G(N)$, every pair $\gamma, \eta \in \mathbb{R}$, and every player $i \in N$, we have

$$
\Phi_{i}(\gamma u+\eta w)=\gamma \Phi_{i}(u)+\eta \Phi_{i}(w)
$$

$\Phi$ is additive if in particular 2.5 holds for $\gamma=\eta=1$.

Axiom 7. Desirability 2 For all $v \in G(N)$ and all $i, j \in N$ if $v(S \cup i) \geq v(S \cup j)$ for all $S \subseteq N \backslash\{i, j\}$, then $\Phi_{i}(v) \geq \Phi_{j}(v)$.

Axiom 8. Null player in a productive environment property: For all $v \in G(N)$ and $i \in N$ such that $i$ is a null player in $v$ and $v(N) \geq 0$, we have $\Phi_{i}(v) \geq 0$.

Axiom 9. Strong differential monotonicity: For all $v, w \in G(N), i, j \in N$, such that $v(S \cup i)-v(S \cup j) \geq$ $w(S \cup i)-w(S \cup j)$ for all $S \subseteq N \backslash\{i, j\}$, we have $\Phi_{i}(v)-\Phi_{j}(v) \geq \Phi_{i}(w)-\Phi_{j}(w)$.

Axiom 10. Positivity: For any $v \in G(N)$ and for all $S, T \subseteq N$ with $T \subseteq S$ such that $v(S) \geq v(T)$, we have $\Phi_{i}(v) \geq 0$.

The most standard characterization of the Shapley value requires efficiency, symmetry, linearity and null player property [15. The ED, on the other hand has been characterized by efficiency, symmetry, linearity and nullifying player property 16 . The characterization theorems of the $\alpha$-Egalitarian Shapley value due to [5] are listed below for later reference.

Theorem 1. (Proposition 1 in [5], p. 59) A value $\Phi$ satisfies efficiency, linearity, symmetry and the null player in a productive environment property if and only if there is an $\alpha \leq 1$ such that $\Phi=\Phi^{\alpha-E S}$.

Theorem 2. (Theorem 2 in [5], p. 59) A value $\Phi$ satisfies efficiency, additivity, desirability and the null player in a productive environment property if and only if there is an $\alpha \in[0,1]$ such that $\Phi=\Phi^{\alpha-E S}$.

Theorem 3. (Theorem 4 in [5], p. 60) For $n \neq 2$, a value $\Phi$ satisfies efficiency, strong differential monotonicity and the null player in a productive environment property if and only if there is an $\alpha \in[0,1]$ such that $\Phi=\Phi^{\alpha-E S}$.

A value that satisfies efficiency, symmetry and linearity is called an ESL value [7. We mention the following proposition from [14] for later reference.

\footnotetext{
${ }^{2}$ In 13 the Desirability axiom is termed as Fair Treatment.
} 
Proposition 1. (Proposition 2 in [14], p. 184) A value $\Phi$ on $G(N)$ is an ESL-value if and only if there exists a unique collection of real constants $B^{\Phi}=\left(\alpha_{s}: s \in\{0,1,2, \ldots, n\}\right)$ with $\alpha_{n}=1$ and $\alpha_{0}=0$ such that for every game $v \in G(N)$,

$$
\Phi_{i}(v)=\sum_{S \subseteq N: i \in S} \frac{(s-1) !(n-s) !}{n !}\left\{\alpha_{s} v(S)-\alpha_{s-1} v(S \backslash i)\right\} .
$$

That is

$$
\Phi_{i}(v)=\Phi_{i}^{S h}\left(B^{\Phi} v\right)
$$

where $\left(B^{\Phi} v\right)(S)=\alpha_{s} v(S)$ for each coalition of size s. This value $\Phi$ is denoted by $\Phi^{E S L}$.

Note that in Proposition 1, $\alpha_{1}=\cdots=\alpha_{n-1}=\alpha$ corresponds to the $\alpha$-Egalitarian Shapley value. We will further use the following lemmata.

Lemma 1. (Lemma 5 in [5], p. 4) Every value $\Phi$ that satisfies efficiency, additivity and desirability also satisfies linearity.

Lemma 2. (Proposition 6 in $[6], p$. 171) For $n \neq 2$, if a value $\Phi$ satisfies efficiency, strong differential monotonicity and null game property then it also satisfies additivity.

\section{A Generalized Egalitarian Shapley value for TU games}

In this section, we propose a Generalized Egalitarian Shapley value for the class $G(N)$ by decomposing it into its subspaces based on the coalition size. Interestingly enough, this class of values incorporates the subclass of all the $\alpha$-Egalitarian Shapley values including the Shapley value and the Equal Division in particular. We proceed as follows: Consider an arbitrary $k$ that ranges over the sizes of the coalitions, namely $\{1,2, \ldots, n\}$. Let us introduce two subspaces of $G(N)$ as follows:

$$
G_{<k}(N)=\{v \in G(N): v(S)=0 \text { for all } s \geq k\} \text { and } G_{\geq k}(N)=\{v \in G(N): v(S)=0 \text { for all } s<k\} .
$$

Then using the standard notation for the direct sum of linear spaces, we get $G(N)=G_{<k}(N) \oplus G_{\geq k}(N)$. It follows that every game $v \in G(N)$ can be written as $v=v_{<k}+v_{\geq k}$ where $v_{<k} \in G_{<k}(N), v_{\geq k} \in G_{\geq k}(N)$ such that $v_{<k}(S)=v(S)$ for all $s<k, v_{<k}(S)=0$ for all $s \geq k$ and $v_{\geq k}(S)=v(S)$ for all $s \geq k$, $v_{\geq k}(S)=0$ for all $s<k$. Consider the following intuitive procedure.

Start with the vector $\boldsymbol{\alpha}=\left(\alpha_{1}, \alpha_{2}\right)$ such that $0 \leq \alpha_{1} \leq \alpha_{2} \leq 1$, a fixed $k$ and apply $\Phi^{\alpha_{1}-E S}$ for $G_{<k}$ and $\Phi^{\alpha_{2}-E S}$ for $G_{\geq k}$. Consider the quantity $\Psi_{k}^{\alpha}$ given by

$$
\Psi_{k}^{\alpha}=\Phi^{\alpha_{1}-E S}\left(v_{<k}\right)+\Phi^{\alpha_{2}-E S}\left(v_{\geq k}\right)
$$

Now, take $1<k_{1}<k_{2} \leq n$ and set $G_{k_{1} \leq,<k_{2}}=\left\{v \in G(N): v(S)=0\right.$ for all $\left.s<k_{1}, s \geq k_{2}\right\}$. It follows that $G(N)=G_{<k_{1}} \oplus G_{k_{1} \leq,<k_{2}} \oplus G_{\geq k_{2}}$. Consequently, every $v \in G(N)$ can be expressed as $v=v_{<k_{1}}+v_{k_{1} \leq,<k_{2}}+v_{\geq k_{2}}$, where

$$
v_{k_{1} \leq,<k_{2}}(S)=\left\{\begin{array}{l}
0, \quad \text { if } s<k_{1} \text { or } s \geq k_{2} \\
v(S), \text { if } k_{1} \leq s<k_{2}
\end{array}\right.
$$

Similarly, by taking $\boldsymbol{\alpha}=\left(\alpha_{1}, \alpha_{2}, \alpha_{3}\right) \in[0,1]^{3}$ where $0 \leq \alpha_{1} \leq \alpha_{2} \leq \alpha_{3} \leq 1$ with $k=\left(k_{1}, k_{2}\right)$, the quantity $\Psi_{k}^{\alpha}$ now looks like:

$$
\Psi_{k}^{\boldsymbol{\alpha}}=\Phi^{\alpha_{1}-E S}\left(v_{<k_{1}}\right)+\Phi^{\alpha_{2}-E S}\left(v_{k_{1} \leq,<k_{2}}\right)+\Phi^{\alpha_{3}-E S}\left(v_{\geq k_{2}}\right) .
$$

Recall that $k$ varies with the size of the coalitions and therefore, taking $k=(1,2, \ldots, n)$, the $n$-tuple with all sizes of the coalitions of $N$, we obtain the following:

$$
G(N)=G_{<2} \oplus G_{2 \leq,<3} \oplus \ldots \oplus G_{n-1 \leq,<n} \oplus G_{\geq n} .
$$


Also, for each $v \in G(N)$ we have,

$$
v=v_{<2}+v_{2 \leq,<3}+\ldots+v_{n-1 \leq,<n}+v_{\geq n} .
$$

Note that, for any $\alpha \in[0,1], \Phi^{\alpha-E S}\left(v_{\geq n}\right)=\Phi^{S h}\left(v_{\geq n}\right)$.

Let $\boldsymbol{\alpha}=\left(\alpha_{1}, \alpha_{2}, \ldots, \alpha_{n}\right) \in[0,1]^{n}$ be such that $0=\alpha_{0} \leq \alpha_{1} \leq \alpha_{2} \leq \ldots \leq \alpha_{n}=1$. The Generalized Egalitarian Shapley value or the $\boldsymbol{\alpha}$-GES value denoted by $\Phi^{\boldsymbol{\alpha}-G E S}$ is then given by,

$$
\Phi_{i}^{\boldsymbol{\alpha}-G E S}(v)=\Phi^{\alpha_{1}-E S}\left(v_{<2}\right)+\sum_{k=2}^{k=n-1} \Phi^{\alpha_{k}-E S}\left(v_{k \leq,<k+1}\right)+\Phi^{\alpha_{n}-E S}\left(v_{\geq n}\right) .
$$

After simplifications, (3.4) can be expressed as follows:

$$
\Phi_{i}^{\boldsymbol{\alpha}-G E S}(v)=\sum_{S: i \in S} \frac{(n-s) !(s-1) !}{n !}\left\{\alpha_{s} v(S)-\alpha_{s-1} v(S \backslash i)\right\}
$$

\subsection{Characterization of the Generalized Egalitarian Shapley value}

Following [5], where the $\alpha$-Egalitarian Shapley value is characterized using efficiency, null player in a productive environment, [(linearity and symmetry) or (additivity and desirability) or strong differential monotonicity] we consider a weaker variation of the null player in a productive environment property to characterize the Generalized Egalitarian Shapley value. This new axiom which we call the Null player in a non-negative environment property is defined as follows.

Axiom 11. Null player in a non-negative environment property: For all $v \in G(N)$ and $i \in N$ such that $i$ is a null player in $v$ and $v(S) \geq 0$ for all $S \subseteq N$, we have $\Phi_{i}(v) \geq 0$.

Remark 1. In view of [5] all the values that combine egalitarianism and marginalism (except the Egalitarian Shapley value) fail to satisfy the null player in a productive environment property, which makes them less appealing. The null player in a productive environment property allows for solidarity with the null player whenever the worth of the grand coalition is non-negative. This, however, makes it a stronger axiom than the null player in a non-negative environment property because the null player in a productive environment property applies even if smaller coalitions generate negative worths. The null player in a non-negative environment property is silent in such cases, and hence it is weaker than the null player in a productive environment property.

Remark 2. The null player in a non-negative environment property is neither weaker nor stronger than positivity: positivity is not weaker because it has implications for all players, not only for null players. The null player in a non-negative environment property is not weaker because it also applies to non-monotonic games that are non-negative.

In the following, we show that the Generalized Egalitarian Shapley value is characterized by efficiency and the null player in a non-negative environment along with [(additivity and desirability) or strong differential monotonicity].

Theorem 4. A value $\Phi$ satisfies efficiency, additivity, desirability and the null player in a non-negative environment property if and only if there exists an $\boldsymbol{\alpha}=\left(\alpha_{1}, \alpha_{2}, \ldots, \alpha_{n}\right) \in[0,1]^{n}$ where $0=\alpha_{0} \leq \alpha_{1} \leq$ $\alpha_{2} \leq \ldots \leq \alpha_{n}=1$ such that $\Phi=\Phi^{\alpha-G E S}$.

Proof. $\Phi^{\alpha-G E S}$ inherits additivity and desirability from $\Phi^{\alpha-E S}$ (see Theorem 2). Again, from 3.4 it is clear that $\Phi^{\boldsymbol{\alpha}-G E S}$ satisfies efficiency and symmetry for $\boldsymbol{\alpha}=\left(\alpha_{1}, \alpha_{2}, \ldots, \alpha_{n}\right) \in[0,1]^{n}$ where $0=\alpha_{0} \leq$ $\alpha_{1} \leq \alpha_{2} \leq \ldots \leq \alpha_{n}=1$. Fix player $i \in N$ and define a game $v \in G(N)$ such that $v(S)=v(S \backslash i)$ for all $\emptyset \neq S \subseteq N$ and $v(S) \geq 0$ for all $S \subseteq N$ containing $i$. Then $i$ is a null player in $v$ and therefore, $v(S \backslash i) \geq 0$ for all $S \subseteq N$ as well. It follows that $\alpha_{s} v(S)-\alpha_{s-1} v(S \backslash i)=\left(\alpha_{s}-\alpha_{s-1}\right) v(S \backslash i) \geq 0$. Thus, $\Phi_{i}^{\boldsymbol{\alpha}-G E S}(v) \geq 0$ and therefore, $\Phi^{\boldsymbol{\alpha}-G E S}$ satisfies the null player in a non-negative environment property. 
Conversely, let $\Phi$ satisfy efficiency, additivity, desirability and the null player in a non-negative environment property. It is easy to check that desirability implies symmetry. By Lemma 1, efficiency, additivity and desirability imply linearity. Consequently, $\Phi$ satisfies symmetry and linearity. Thus, $\Phi$ is an ESL value. It follows from Proposition 1 that there exists an $\boldsymbol{\alpha}=\left(\alpha_{0}, \alpha_{1}, \ldots, \alpha_{n}\right) \in \mathbb{R}^{n}$ with $\alpha_{0}=0, \alpha_{n}=1$ such that

$$
\Phi_{i}(v)=\sum_{S: i \in S} \frac{(n-s) !(s-1) !}{n !}\left\{\alpha_{s} v(S)-\alpha_{s-1} v(S \backslash i)\right\} .
$$

We need to show that $0=\alpha_{0} \leq \alpha_{1} \leq \alpha_{2} \leq \ldots \leq \alpha_{n}=1$. Fix $i \in N$ and $S \subseteq N$ such that $i \in S$ and $s>1$. Define the game $w \in G(N)$ by $w=e_{S}+e_{S \backslash i}$. Then $i$ is a null player in $w$ and $w(K) \geq 0$ for all $K \subseteq N$. Therefore by the null player in a non-negative environment property, $\Phi_{i}(w) \geq 0$. Since $\Phi_{i}(w)=\frac{(n-s) !(s-1) !}{n !}\left\{\alpha_{s}-\alpha_{s-1}\right\}$, we have $\alpha_{s} \geq \alpha_{s-1}$ for all $1<s \leq n$. It remains to show that $\alpha_{1} \geq \alpha_{0}=0$. By efficiency and symmetry, we have $\Phi_{i}\left(v_{0}\right)=0$ for all $i \in N$. Using desirability, we get $\Phi_{1}\left(e_{\{1\}}\right) \geq \Phi_{1}\left(v_{0}\right)$. It follows that $\Phi_{1}\left(e_{\{1\}}\right) \geq 0$. Since $\Phi_{1}\left(e_{\{1\}}\right)=\frac{\alpha_{1}}{n}$, we have $\alpha_{1} \geq 0$. This completes the proof.

Our next characterization of the $\boldsymbol{\alpha}$-GES value without additivity relies on the results of $[5,6$.

Theorem 5. For $n \neq 2$, a value $\Phi$ satisfies efficiency, strong differential monotonicity and the null player in a non-negative environment property if and only if there exists an $\boldsymbol{\alpha}=\left(\alpha_{1}, \alpha_{2}, \ldots, \alpha_{n}\right) \in[0,1]^{n}$ where $0=\alpha_{0} \leq \alpha_{1} \leq \alpha_{2} \leq \ldots \leq \alpha_{n}=1$ such that $\Phi=\Phi^{\alpha-G E S}$.

Proof. $\Phi^{\boldsymbol{\alpha}-G E S}$ satisfies efficiency and the null player in a non-negative environment property by Theorem 4. Moreover, $\Phi^{\alpha-G E S}$ inherits strong differential monotonicity from $\Phi^{\alpha-E S}$.

Let the value $\Phi$ satisfy efficiency, the null player in a non-negative environment property and strong differential monotonicity.

For $n=1, \Phi^{\boldsymbol{\alpha}-G E S}=\Phi^{\alpha-E S}$ and therefore, the result follows from Theorem 3

Let now $n>2$. From efficiency and the null player in a non-negative environment property, $\Phi_{i}\left(v_{0}\right)=0$ for each $i \in N$, i.e., $\Phi$ satisfies the null game property. Clearly the null game property and strong differential monotonicity imply desirability. In view of Lemma 2, $\Phi$ satisfies additivity. Now, the result follows from Theorem 4 .

Remark 3. In [5], an example is given to show that Theorem 3 fails for $n=2$. We build a similar example here. Let $N=\{1,2\}$, and define the value $\Phi$ on $N$ by

$$
\Phi_{i}(v)=\frac{v(N)}{2}+v(i) \cdot 2^{-|v(i)|-2}-v(j) \cdot 2^{-|v(j)|-2}
$$

for all $i, j \in N, i \neq j$, and for all $v \in G(\{1,2\})$. Then $\Phi$ satisfies efficiency, strong differential monotonicity and the null player in a non-negative environment property but not additivity. It follows that there does not exist any $\boldsymbol{\alpha} \in[0,1]^{n}$ such that $\Phi=\Phi^{\boldsymbol{\alpha}-G E S}$.

\subsection{Comparison with existing solutions}

The $\boldsymbol{\alpha}$-GES values generalize the Egalitarian Shapley values due to [10] and belong to the class of ESL values. In [13, it is shown that among the ESL values, desirability and positivity are satisfied if and only if $0 \leq \alpha_{s} \leq 1$ for $1 \leq s \leq n-1$ in the expression (2.6). In view of (3.5), it is clear that this subclass of ESL value contains the $\boldsymbol{\alpha}$-GES values, which therefore satisfy positivity and desirability. It is also interesting to note that the class of $\boldsymbol{\alpha}$-GES values includes the class of discounted Shapley values due to [10. 羟

\footnotetext{
${ }^{3}$ For $\delta \in[0,1]$ the $\delta$-discounted Shapley value $\Phi^{\delta}: G(N) \rightarrow \mathbb{R}^{n}$ is given by

$$
\Phi^{\delta}(v)=\sum_{S \subseteq N: i \in S} \frac{(n-s) !(s-1) !}{n !} \delta^{n-s}\{v(S)-\delta v(S \backslash i)\}
$$

Finally, note that the class of $\boldsymbol{\alpha}$-GES values does not include the Solidarity value [12, which violates the null player in a non-negative environment property.

${ }^{4}$ Solidarity value is given by 2.6 with $\alpha_{s}=\frac{1}{(s+1)}$ for $1<s<n$.
} 


\section{Conclusion}

We have obtained a Generalized version of the Egalitarian Shapley value and its characterizations based on some standard axioms. There exist possibilities for exploring alternative characterizations in the line of [4] and for studying the implementation mechanism for the class of Generalized Egalitarian Shapley values. We leave this for future research.

\section{Acknowledgement}

The authors would like to thank the associate editor and the three anonymous referees for their comments and suggestions. This research was funded by UKIERI[184-15/2017(IC)].

\section{References}

[1] E. Algaba, Fragnelli, V. and Sánchez-Soriano, J. (2019) Handbook of the Shapley Value. CRC Press, Taylor \& Francis Group. USA.

[2] Béal, S., Rémila, E. and Solal, P. (2017) A strategic implementation of the sequential equal surplus division rule for digraph cooperative games, Annals of Operations Research, 253, 43-59. https://doi.org/10.1007/s10479-016-2290-5.

[3] Borkotokey, S., Choudhary, D., Gogoi, L. and Kumar, R. (2020) Group Interactions in TU games : The k-lateral value, European Journal of Operational Research, DOI: https://doi.org/10.1016/j.ejor.2020.03.054.

[4] Casajus, A. and Huettner, F. (2014) Weakly monotonic solutions for cooperative games, Journal of Economic Theory, 154, 162-172.

[5] Casajus, A. and Huettner, F. (2013) Null players, solidarity, and the Egalitarian Shapley values, Journal of Mathematical Economics, 49, 58-61.

[6] Casajus, A. (2011) Differential marginality, van den Brink fairness, and the Shapley value, Theory and Decision, 71, 163-174.

[7] Driessen, T., and Radzik, T., (2003) Extensions of Hart and Mas-Colells consistency to efficient, linear, and symmetric values for TU-games. In: Petrosyan, L.A., Yeung, D.W.K. (Eds.), ICM Millennium Lectures on Games. Springer-Verlag, Heidelberg, Germany, 147-166.

[8] Fehr E., Bernhard H. and Rockenbach B., (2008) Egalitarianism in young children, Nature, 454, 1079 -1083. DOI:10.1038/nature07155.

[9] He, Q., Pan, Y., and Sarangi, S., (2018) Lineage-based Heterogeneity and Cooperative Behavior in Rural China, Journal of Comparative Economics, 46, 248-269.

[10] Joosten, R., (1996) Dynamics, equilibria and values dissertation. Maastricht University.

[11] Kalai, E., Samet, D., (1987) On weighted Shapley values. International Journal of Game Theory, 16, 205-222.

[12] Nowak, A. S. and Radzik, T. (1994) A solidarity value for n-person transferable utility games, International Journal of Game Theory, 23, 43-48.

[13] Radzik, T., and Driessen, T., (2013) On a family of values for TU-games generalizing the Shapley value, Mathematical Social Sciences, 65, 105-111.

[14] Radzik, T., Driessen, T., (2016) Modeling values for TU-games using generalized versions of consistency, standardness, and the null player property, Mathematical Methods of Operations Research, 83, 179-205. 
[15] Shapley, L. S. (1953) A value for n-person games, in Kuhn, H. and Tucker, A.W. (eds.), Contribution to the Theory of games II, Princeton, New Jersey, Princeton University Press, 307-317.

[16] van den Brink, R. (2007) Null or nullifying players: the difference between the Shapley value and equal division solutions, Journal of Economic Theory, 136, 767-775.

[17] van den Brink, R., Funaki, Y., and Ju, Y., (2013) Reconciling marginalism with egalitarianism: consistency, monotonicity, and implementation of egalitarian Shapley values, Social Choice and Welfare, 40, 693-714.

[18] Yokote, K. and Funaki, Y., (2017) Monotonicity implies linearity: characterizations of convex combinations of solutions to cooperative games, Social Choice and Welfare, 49, 171-203. 